\title{
Nouvelle économie du narcissisme
}

\section{Colette Soler}

Ne croyez pas que mon titre provienne du fait j'ai attrapé le virus de la nouveauté à tout prix qui caractérise notre époque, ce n'est pas du tout le cas, je n'aime pas vraiment cette épidémie. Ce que je suis en train de travailler sur ce thème provient de deux sources. D'abord le sentiment, que j'ai depuis longtemps, que ce que l'on a retenu de l'enseignement de Lacan qui provient essentiellement d'avant les années 1965, ne permet ni de penser ni de faire face aux évolutions de l'époque à tous les niveaux, celui des mœurs, des structures sociales, des instruments techno-scientifiques. Autrement dit, le sentiment que les instruments théoriques de la pratique des analystes aujourd'hui ne sont pas à l'heure de ce début de siècle. Mais pas non plus plus à l'heure de Lacan, car comment ignorer que lui-même a opéré des changements de perspectives considérables ? Ce changement est majeur concernant le narcissisme. Il l'a mis d'abord au compte de l'imaginaire, c'est notoire, mais d'un imaginaire subordonné à la chaîne du symbolique. Mais à partir de 1973, à longueur de leçons, il serine que les trois consistances - Imaginaire, Symbolique et Réel - sont équivalentes et non subordonnées l'une à l'autre, comme il l'avait d'abord affirmé. Eh bien, j'en conclus non seulement qu'il faut saisir les raisons de ce changement qui n'est pas arbitraire, mais surtout qu'il faut repenser, en tous cas mettre à jour, tout ce qu'il a développé sur la base de cette première thèse d'un Symbolique langagier qui ordonne, et donc subordonne, tout ce qui se présente dans l'imaginaire, au premier rang de quoi se placent et le narcissisme et la consistance imaginaire du Moi, en contraste avec la division propre au sujet du signifiant. Regardez les schémas $L$ et $R$, le texte sur Schebert, et le graphe du désir, c'est limpide, ils déplient tous, visualisent et topologisent ce postulat de ce que j'avais appelé naguère, en suivant ces élaborations, «l'image serve " d'un symbolique souverain. De fait, après Fonction et champ de la parole et $d u$ langage en psychanalyse, Lacan (1953/1966) a subordonné l'imaginaire du miroir au symbolique du langage, tout comme le signifié est subordonné au signifiant. Du coup, il a souligné que la présence de l'Autre, grand A, conditionne même le fait que l'enfant se reconnaisse et s'aime dans son image. Quand, avec le nœud borroméen, il se ravise, il récuse cette subordination quand il martèle que les trois consistances sont autonomes et équivalentes, comment ne pas se demander ce que ça change sur le plan clinique et analytique?

Voyez l'enjeu. Avant tout d'examen, on peut poser la question: comment, si l'imaginaire n'est pas subordonné, peut-on continuer à penser que le narcissisme du moi soit réductible par le symbolique, et qu'une analyse, en construisant le 
sujet divisé du signifiant, réduise les prétentions narcissiques ? L’enjeu analytique est de taille, aussi bien que la conception que l'on se fait de l'homme, que Lacan écrira LOM, à partir de son hypothèse qui définit la structure - non la structure de langage, mais la structure comme « l'effet de langage »sur le vivant. Qu'est-ce que ça change, d'abord, à la conception de ce qui est au cœur de l'imaginaire et dont il est parti, à savoir la fonction du miroir, et ensuite au champ des significations qui appartiennent au registre de l'imaginaire ordonné par le symbolique comme le signifié est subordonné au signifiant ? Qu'est-ce qu'un imaginaire autonome, et quelle est la différence entre subordination et nouage éventuel dans le nœud borroméen?

\section{L'enjeu du miroir}

L'enjeu pour Lacan et pour le sujet. Je pars du début, et de l'évidence : le miroir est premier. Pour marquer les bornes du parcours de Lacan, je souligne immédiatement que cette formule, je la construit sur le modèle d'une autre, qui dit dans le Conférence sur Joyce (LACAN, 1975a/2001), l'SKbeau est premier.

Dans l'enseignement de Lacan non seulement le stade du miroir se place dans ses antécédents, mais, en outre, selon sa thèse, pour l'enfant, il est également premier. Il est même, j'y reviendrai, d'avant le sujet.

Après la thèse sur Aimé et sa psychose, Le stade du miroir (LACAN, 1949/1966) est son texte majeur, jamais remis en cause, quoique complété et remanié - je laisse de côté le texte sur la famille (LACAN, 1938/2001) qui lui avait été demandé par Henri Vallon. Or, je remarque que dans ce texte du stade du miroir, manquent deux grandes références que l'on pourrait y attendre: ni Narcisse, celui du mythe, ni surtout Freud, ne sont évoqués. Qu'est-ce que ça indique ? Ça nous met, je pense, sur la piste de la question implicite qui sous-tend le texte de Lacan. Pas de lecture d'un texte théorique, qu'il soit de philosophie ou de psychanalyse, qui n'ait à extraire la question à laquelle le texte s'attaque. Pour introduire le narcissisme, de Freud (1914/1985), tout comme le mythe de Narcisse, d'ailleurs s'attaquaient à la question du placement de la libido érotique, ce que l'on nomme investissement des objets, ou relation d'objet, notion célèbre dans la psychanalyse à l'arrivée de Lacan, d'ailleurs. La question sous-jacente au stade du miroir est un peu décalée par rapport à cette problématique, et elle est plus large. Certes, dans ce stade, l'image devient le premier objet, on peut donc y voir un stade de la libido, mais cet amour de l'image est déterminée par autre chose, à savoir sa fonction identitaire: elle est constituante d'une première strate de l'identité. De là on peut dire ce qu'est la question fondamentale, implicite, à laquelle répond le stade du miroir : elle est de savoir comment l'enfant d'homme, qui est un petit organisme, un petit animal, devient un humain socialisé et socialisable. C'est une autre question que celle de Freud, qui présuppose l'humanité de l'enfant comme donnée. Mais c'était aussi la 
question des psychologues de l'époque, Wallon notamment, qui avait demandé à Lacan son article sur la famille, tous étaient préoccupés au fond par l'avènement à l'humain socialisé ou socialisable proprement dit. D'où l'intérêt passionné pour les enfants sauvages. Lacan est sur cet axe, qui est de son temps.

L'identification est là reconnue comme l'instrument premier de la socialisation.

Qu'est-ce qui fonde l'importance, voire la nécessité, de cette première identification chez le petit d'homme? Il fallait bien que Lacan se pose cette question, puisque l'image y a une fonction autre que chez l'animal, qui ne touche apparemment ni à la survie ni à la reproduction. Dans sa thèse la plus générale et la plus connue, mais postérieure, Lacan répond : l'identification est nécessitée par l'effet manque à être du sujet que produit le langage. Mais pour le petit, qui n'use pas encore de lalangue - le langage c'est ça, l'usage de lalangue -, ça ne peut pas être le cas. Et Lacan de chercher une autre cause et de se référer à une cause réelle, à savoir aux effets de la prématuration de la naissance chez l'animal humain, avec le morcellement des fonctions vitales qui s'ensuit durant les premières années, et qu'il suppose produire un vécu « d'insuffisance ", que l'identification au Un de l'image, " orthopédique de sa totalité » (LACAN, 1949/1966, p. 100), résoudrait, en anticipant la solution qui ne viendra en fait que par la maturation du système nerveux. À vrai dire, rien n'indique ce malaise chez le petit, c'est plutôt le contraire. Freud (1914/1985) est plus convainquant quand au tout début de Pour introduire le narcissisme, il pose que ce qui précède l'unité du moi, c'est non pas un douloureux sentiment d'insuffisance répercutant l'immaturité des fonctions adaptatives, mais un auto-érotisme que l'on peut dire heureux, satisfait, le plaisir prélevé sur le corps propre en dérivation des fonctions vitales, multiples certes, mais dont le morcellement n'est pas synonyme de malaise vital tant que la demande de l'Autre ne s'en mêle pas. La psychanalyse rencontre certes les fantasmes et angoisses du corps morcelé, mais chez des analysants adultes ou enfants dont l'unité du moi est déjà établie. On ne voit pas d'ailleurs comment il pourrait y avoir une conscience de morcellement sans une conscience d'unité, puisqu'elles sont relatives l'une à l'autre. Lacan d'ailleurs n'a pas manquéplus tard de railler sa construction et de se gausser de son appel à la prématuration, au moment où il avait mis en évidence le vrai principe du corps morcelé, qui n'est pas la prématuration, mais le signifiant.

L’identité par identification, est évidemmentune identité aliénée faite d'un premier semblant. Lacan aura-t-il suffisamment insisté sur ce trait de l'aliénation à l'image et de l'aspiration correspondante à s'en libérer de cette aliénation, avec l'espoir que ce soit possible. Cet espoir qui a sévi dans la psychanalyse lacanienne dès le début, encouragée par Lacan construisant, son opposition entre la gonfle narcissique du moi et le sujet divisé du signifiant.

Notez qu'avec cette identification scopique, le projeteur de Lacan est dirigé, curieusement, sur ce qu'il y a de plus étranger à l'inconscient, à savoir le registre de ce qui se voit. Il fallait bien que Lacan ait une autre question pressante pour qu'il 
en soit ainsi. Il précise que cette image est «le seuil du visible», qui ouvre donc le registre scopique, noyau de tout paraître. Là, il s'agit bien de voir, et il emprunte non à Freud, mais à l'éthologie qui a établi ce qui n'est nullement un mythe, mais une fonction vitale, bien réelle, de l'image visuellement perçue chez l'animal. Quelle est cette fonction chez l'animal? D’abord,une fonction de transmission entre les générations animales de rien moins que le savoir instinctuel. Ce savoir instinctuel opère sur deux plans. Il est nécessaire d'abord pourla survie - le poussin ne picore que s'il a vu la poule picorer -, ensuite il intervient dans ce qui assure la reproduction de l'espèce, qui ne va pas sans les rituels de la parade visuelle, et l'épinoche ne se reproduit pas sans une image de l'espèce, mais la sienne dans le miroir fait aussi bien que celle d'un congénère. Pour l'enfant, à la différence de l'animal, l'image ne sert ni la survie, qui est assurée par l'Autre du fait de la prématuration, ni le sexe, qui ne vient que plus tard. Sa fonction est d'identification, elle offre le noyau de l'amour de soi, lidido et identité fusionnant à cette occasion sur l'image du corps propre. Et ceci bien avant toute problématique sexuée. Elle précède diachroniquement non lalangue, qui est un bain d'origine, mais l'acquisition du langage. Il s'agit de l'enfant qui ne parle pas encore, Lacan le souligne.

Rien dans tout ça qui évoque l'inconscient, c'est tout de même frappant ! C'est dans les suites de ce stade du miroir, dans un deuxième temps, que dans La causalité psychique, Lacan (1949/1966) repense l'inconscient freudien. Il le fait à partir de cette fonction de l'image, seuil du monde visible, préalablement avérée expérimentalement par l'observation, pas par la parole - et l'observation, c'est un autre point d'ancrage dans la pensée scientifique que celui de Freud, c'est l'ancrage expérimental - et il avance un inconscient-imago, fait d'imago originaires. Lesquelles celles des premières relations expérimentées dans le contexte des premières années, un inconscient fait des premières marques sociales donc, en outre un set d'images fixes, qui, par leur fixité, sont d'ailleurs déjà voisines du signifiant.

Cette mise en suspens de la question de l'inconscient se conçoit, car dans cette phase du miroir, le sujet n'a pas encore fait " entrée dans le Réel » (LACAN, 1960b/1996, p. 655). Il s'agit d'une phase d'avant le sujet. L'expression d'avant le sujet peut surprendre, car nous sommes habitués à direavec Lacan que, avant même de naître, le petit d'homme est sujet pour l'Autre. Oui il «fait sujet dans le dire des parents» (LACAN, 1972/2001, p. 460), et c'est un dommage à priori dit Lacan dans L'étourdit. A priori bien sûr, puisque les effets dommageables de ce dire sont programmés d'avant même que le petit ne vienne à l'existence, et indépendamment de ce que seront ses caractéristiques propres, notamment quant au sexe, selon qu'il sera garçon ou fille. Qu'il naisse hermaphrodite pour voir, s'exclame Lacan! Cependant, il faut faire la différence entre être sujet dans le dire de l'Autre et être sujet « dans le réel ». Je cite : «Il faut qu'au besoin [...] s'ajoute la demande pour que le sujet [...] fasse son entrée dans le réel, cependant que le besoin devient pulsion » (LACAN, 1960b/1996, p. 655). 
Il n'entre dans le réel, i.e. ne sort de l'Autre, qu'avec la demande articulée comme première forme d'un dynamisme libidinale induit non par l'image spéculaire du transitivisme, mais par le langage générateur à la fois du sujet et des pulsions. Le transitivisme, lui, est plus une confusion des images qu'un ordre entre les images, et c'est le langage évidemment qui va infuser rétroactivement dans l'image le statut du un différentiel propre au signifiant.

\section{Un autre narcissisme}

Quelle est, ou quelles sont, le(s) fonction(s) pour le sujet de ce narcissisme de l'image tel qu'on l'aperçoit dans cette première élaboration? On m'a parlé d'une très jolie phrase d'Oscar Wilde, disant « l'amour de soi est un amour qui dure toute la vie »(WILDE, 1993, p. 239), plus fiable donc que les autres. Ça vaut la peine de s'y arrêter.

La première que j'ai soulignée est une fonction identitaire. L'enfant se reconnaît dans cette image. Évidemment, toute fonction identitaire suppose du Un, ici c'est le un de la gestalt de l'image, précédant le un du signifiant. Alors, le narcissisme, si on doit donner une définition simple, c'est, dès le mythe, l'amour de soi-même, d'un soi-même identifié par l'unité de cette image, indépendamment de ses autres caractéristiques, et notamment de sa beauté, seule compte son unité gestaltique dans l'accent mis par Lacan.

Comme tout amour, il comporte une dimension d'idéalisation, qui va vers ces variantes de l'idéalisation, que sont la surévaluation, la vanité, l'infatuation. Il "se croit », le petit narcisse. Ça frôle parfois la folie, le délire mégalomaniaque, c'est son côté dérisoire et à chaque fois surprenant quand on constate combien, par exemple, l'auto-évaluation satisfaite peut se cacher, même derrière ce qui se présente comme un manque de confiance en soi, spécialement chez les femmes. Je n'insiste pas plus, c'est un des ressorts tragicomiques de la vie sociale, mais je veux souligner autre chose.

D'abord, que le narcissisme de cette phase, c'est de l'amour, pas du désir, pas de la pulsion, et l'introduction de ces deux dimensions dans l'expérience obligera Lacan à repenser ou à complexifier la notion. J'ai marqué la différence avec Freud qui, lui, dès le départ, place le narcissisme au niveau d'un avatar du désir sexuel et des pulsions. Mais Lacan l'a dit, quand on aime il n'est pas question de sexe. C'est donc au fond un soi-même encore très incomplet le narcissisme du miroir, de l'amour de son image, car il y a en chacun quelque chose qui se préfère à son image.

J'ai dit que la question de Lacan venant de son travail sur les psychotiques, était celle de la socialisation de l'enfant. Or dès1949, Lacan fait de l'identification narcissique, paradoxalement par rapport à l'idée que l'on s'en fait, la matrice de la première fonction socialisante, on ne le souligne pas assez. Il a établit une continuité 
entre l'image du corps propre et l'image du semblable, c'est connu, et la fonction qu'il attribue à l'identification transférée au semblable, est explicite. Ecrits (LACAN, 1949/1966, p. 101): j'abrège les citations « le stade du miroir inaugure par l'identification à l'imago du semblable [...] la dialectique qui, dès lors lie le je à des situations socialement élaborées ", et il évoque ensuite " une médiation par le désir de l'autre » sans majuscule, médiation constituante des objets du désir. C’était déjà prêter au transitivisme narcissique une fonction qui va bien au-delà de la contemplation de l'image, qui y inclut le désir même, en tout cas les appétences des parlants, qui en un mot fait paradoxalement du narcissisme un des principes du social.

Je suis frappée cependant du contraste avec le mythe, qui ne dit pas tout à fait la même chose que la psychanalyse. En effet, dès les premiers pas du mythe d'où vient le terme, Narcisse se situe d'être, hors relation. On connaît le noyau du l'hystoire rapporté par Ovide. Narcisse (1992) au départ, c'est le chasseur solitaire, indifférent, totalement insensible aux charmes des nymphes que sa beauté captive, notamment à ceux de la nymphe Écho. On pourrait gloser sur son nom qui fait d'elle un reflet sonore et pas visuel. Narcisse donc est un autosuffisant, aujourd'hui on dirait a narcissistic personality, Freud dirait peut-être narcissisme primaire, jusqu'à ce que Némésis, la vengeance, ne lui fasse, pour son malheur, rencontrer son propre reflet dans l'eau, qui, dès lors, le captive et devient son unique objet. Le voilà donc entré enrelation avec l'image qui entame son autosuffisance, et qui est un objet mortellement inaccessible. Vous voyez l'inversion.

Par contre, ce que le mythe implique, c'est que l'on n'a pas attendu la psychanalyse pour percevoir qu'il y a du mortifère dans l'image, mais surtout savoir que la position hétérosexuelle de la libido est sujette à des ratés. Puisqu'au fond avec la faute de Narcisse dans le mythe, c'est dans sa non relation à l'autre sexe, et pas au semblable qui, lui, n'est pas sexué. Le mythe, avec cette rencontre de l'image du corps propre, illustre au fond ce que je peux appeler une sorte de malédiction de l'image, mais, plus encore, une malédiction de la relation. Pour Narcisse cette image libidinalisée se substitue à la proie qui était jusque-là la cause, voire l'objet de son désir de chasseur, désir séparateur, qui le soustrayait de la libido hétérosexuelle, qui donc le mettait à l'abri de l'autre malédiction, celle sur le sexe. Le stade du miroir de Lacan relaye en partie cette dimension de malédiction de l'image, mais positive, par contre, sa fonction socialisante de relation au semblable. Ce décalage ne peut pas être sans rapport avec les menaces qui pesaient déjà sur les liens sociaux à l'époque de Lacan, bien autrement qu'à l'époque de la cité grecque.

Aujourd'hui le narcissisme de l'image a pris une dimension inimaginable, et inimaginablement active, par rapport au passé même récent. On assiste à une véritable culture de l'image - pensez à la pratique des selfie (on a le miroir dans la poche), et toutes les techniques actuelles de fabrication des corps imaginaires, avec les normes en usage de la silhouette d'abord, avec l'industrie de la mode qui 
le recouvre, de la chirurgie esthétique qui le transforme, mais aussi de la nourriture qui lui donne son volume. Sans oublier les pratiques de marquage à visée distinctive, qui vont des tatouages jusqu'au body art. On n'en finirait pas d'énumérer, avec les nouveaux pouvoirs de manipulation de l'image dûs à la technique, la valeur nouvelle que le sujet d'aujourd'hui confère à leur image prise comme un indexe d'identité. Une identité qui se montre, qui s'offre à être vue, en marge donc de ce qui ne peut pas se voir du sujet. Les psychanalystes, qui ont affaire aux sujets en tant qu'ils parlent et pas en tant qu'ils se montrent, ont tendance à dénoncer ces nouveaux faits de civilisation. Pourtant, il ne faut pas oublier que, de toujours, les paradoxes de l'identité ont trouvé leur ressort, pour l'essentiel, dans la disjonction entre l'être réel, et le paraître, le paraître qui se dédouble entre le paraître de l'image, le paraître photographique en quelque sorte, et ce qui apparaît de non-photographique dans la signification par le biais symbolique, à savoir des idéaux que Freud dit du moi, et que Lacan dit de l'Autre majuscule, grand I de grand A, I(A) qui décident, entre autre chose, de la valeur des images. Voir le graphe du désir. Cette disjonction du réel et du paraître n'est pas une découverte de la psychanalyse, et pas non plus aliénation des individus dans le paraître. « Je est un autre ", la formule est connue d'avant. Et n'est-ce pas un grand thème de l'analysant ? D’un côté, il s'interroge pour détecter comment il est vu, ce qu'on lui « renvoie » comme il dit, et de l'autre, il proteste, « je ne suis pas ce que vous croyez " - là encore, j'emprunte au discours commun à la petite histoire de celle que l'on prend pour une coquette et qui s'insurge, s'inscrivant donc en faux, mais qui, d'un autre côté, s'évertue pour coïncider avec l'image idéale qui, pourtant, lui donne le sentiment d'être dépossédée d'elle-même.

Il y a donc pas seulement ce qui se voit, mais ce qui ne se voit pas, à savoir comment l'autre me voit, et j'ai mis l'accent sur le fait que Lacan utilise, en 1960, l'expression «narcissisme du désir» distinct du "narcissisme de l'ego ", qui est, dit-il, son prototype. C'est dans Propos pour un congrès sur la sexualité féminine (LACAN, 1960a/1996). Qu'est-ce qu'un narcissisme du désir ? C'est un narcissisme qui, comme celui de l'ego, a une fonction identitaire, c'est pourquoi celui-ci en est le prototype, mais avec le désir on introduit le registre du sexe où justement manque ce qui ferait l'identité homme/femme. Ce narcissisme du désir, il l'introduit dans un paragraphesur les femmes, où il pose que ce qui détermine la frigidité, l'identification à «l'étalon phallique ». Ça consiste à ériger, au niveau du paraître, le signification du phallus, qui de sa nature est refoulé, et dont le refoulement a pour effet de projeter toutes les manifestations du sexe dans le paraître. Ce n'est pas le paraître de la simple image scopique, du selfie, c'est la paraître de la parade et de la mascarade, tout le jeu de la comédie des sexes, pour convoquer, dans le paraître, ce qui ne se voit pas. Ce qui y fait unité identifiante au final, ce n'est pas l'image-là, mais un signifiant, le phallus, signifiant du manque, et, avec 
lui, un imaginaire élargi jusqu'à inclure, outre la forme du corps, l'ensemble des significations du sexe dans le discours. Le miroir n'est plus alors simplement une surface physique propre à renvoyer un reflet visuel, c'est, comme je m'étais exprimée naguère, un « miroir parlant ». Celui que Lacan figure dans son schéma optique. «Miroir, dis-moi si je suis la plus belle?».Un miroir supposé savoir, donc. Supposé savoir, bien sûr, ce qui échappe à ma vue, mais, plus largement, ce qui échappe à toute vue possible et qui n'est pas du registre de la vision, et qui est de savoir comment l'Autre me regarde, autrement dit, que suis-je pour lui ? Là, s'ouvre un autre chapitre des malheurs de Narcisse: celui de sa non suffisance.

\section{Les malheurs de Narcisse}

Narcisse, il est loin d'être autosuffisant, il est à la merci du miroir, sous le double aspect que j'ai dit. Aux deux niveaux de l'image qui se montre, que du désir qui ne se voit pas, ne s'énonce pas, mais qui n'apparaît pas moins, car il s'entend dans la parole, et se perçoit dans l'action. Le miroir qu'est l'Autre présentifié par les autres, met paradoxalement le petit narcisse du stade du miroir à la merci de l'inconnu, car il subordonne la relation à l'image primaire, à la relation à l'Autre barré, dont Saint Augustin, dans sa fameuse phrase, avait déjà pris la dimension. Il faudrait donc ouvrir le chapître des malheurs de ce Narcisse qui, aliéné déjà à une image qui n'est pas lui, est en outre paradoxalement enchaîné à un regard hétérotopique - bien loin d'être autosuffisant. Ce regard peut être de partout et de nulle part, car que serait une image que nul ne verrait ou une signification qui ne serait pour personne? Pas étonnant que l'on ait inventé un dieu qui voit tout, les images et leur au-delà de signification et de sens. Pas étonnant non plus que l'on rêve parfois, à l'inverse, du manteau d'invisibilité, fantasme sans doute propice au voyeur et autres ruses des perversités, mais propice d'abord à la soustraction qui libèrerait. Pas étonnant, enfin, que l'on se batte, que l'on s'évertue pour s'assurer de la possession d'une image qui ne dépend pas de moi, car sa valeur vient l'Autre, et dont je peux être dépossédé, et qui donc fait passion, entre autre, envie et jalousie. Pas étonnant, enfin, et plus essentiel, que l'on aspire à ce qui permettrait une séparation. Toute la question étant de savoir s'il y a un narcissisme de séparation possible, et quel serait son instrument si ce n'est ni l'image, ni le désir qui, eux, assujettissent à l'autre, sans majuscule, ou à l'Autre, avec majuscule.

\section{Narcisse et autrui}

En effet à quelle relation au semblable préside le narcissisme dont j'ai souligné qu'il est la matrice première du social, à quel relation préside-t-il dans son aspiration à, disons «se faire beau »? Beau pour l'œil de quelque autre, sans majus- 
cule. C'est la tactique du «m'as-tu-vu»? Cette formule, avec ces deux pronoms personnels, dont Lacan a tellement fait cas, inclut la nécessaire dimension relationnelle du narcissisme, et indique bien la dépendance de Narcisse. Le selfie et remarquable à cet égard. Le sujet se trouve assez intéressant pour se mirer dans un vrai miroir, mais il lui faut ensuite envoyer le selfie à quelques autres pour se mirer dans l'œil de ces autres. Sur l'île déserte le selfie ne serait pas d'un grand secours, on le pressent, et c'est pourquoi on ne dit pas «n'oubliez pas votre miroir», mais on demande " quel livre emporteriez vous sur l'île? », parce que le miroir de l'Autre est un miroir verbal, qui n'exige pas la présence du corps. Pas de Narcisse hors d'une relation de séduction en tous cas. Le personnage du séducteur ou de la séductrice n'a pas bonne presse, mais c'est parce qu'on confond avec l'infidèle, Don Juan, ou l'aguicheuse. Pourtant, c'est une forme de la demande la séduction, et l'analysant n'y échappe pas; loin de là, dans sa parole transférentielle, que fait-il, sinon " manœuvrer » le miroir de l'Autre pour paraître aimable et se trouver aimable du même coup. Quoi de mieux que l'expérience du transfert pour s'assurer que l'amour de soi se soutient de l'amour reçu de l'Autre?

Du coup, avec l'autre pan de la relation à autrui c'est... la compétition. « Miroir, dit-moi si je suis la plus belle», parce qu'il ne suffit pas d'être belle, mais la plus belle. Encore, n'est-ce pas le propre des femmes. À ce niveau, les passions primaires font rage, l'envie qui enrage de recevoir moins, encore Saint Augustin, la jalousie qui fulmine et se morfond d'être exclue d'un lien d'amour, réel ou supposé, la rivalité qui combat avec la volonté de l'emporter. Je trouve assez inutile d'en faire des gorges chaudes et de déplorer ces vilaines passions, la religion chrétienne y suffit, pas besoin de la psychanalyse pour ça. Par contre, on ne devrait pas oublier que la forme la plus éminente de la compétition, c'est l'émulation dans laquelle l'affirmation de soi est moins destructrice de l'autre que... productrice, puisqu'il en sort les œuvres de la civilisation, ces productions qui émerveillaient tellement Freud, qu'il a rebaptisé cette fécondité du terme de sublimation. Lacan, à la fin de son enseignement, en a rabattu sur cette sublimation, et d'un mot : l'escabeau.

\section{Un autre narcissisme}

L'escabeau redéfinit le narcissisme, c'est l'instrument d'un narcissisme actif, combattant et producteur. C'est, donc, plus que de l'amour de soi, c'est l'affirmation de soi, éventuellement par le biais de l'offre à la civilisation. Dit plus à ras de l'expérience, l'escabeau, c'est ce avec quoi chacun tente de se faire valoir pour séduire l'œil de l'autre. Que l'image soit le premier véhicule de cette identification narcissique indique pour l'humain une prévalence du visible, dont la question est de savoir jusqu'où elle s'égale à la prévalence du sonore du langage. Dans la diachronique des dites phases du développement, nous nous sommes habitués 
à ordonner les registres pulsionnels, qui apparaissent en même temps que le sujet dans le réel, en commençant par l'oral et l'anal, qui correspondent aux deux objets de la demande de l'Autre, et ensuite le regard et la voix, objets du désir de l'Autre dont sa présence fait signe. Mais, au fond, voix et regard sont là d'origine, ils ne suivent pas diachroniquement le nourrissage et l'éducation des sphincters; l'enfant en est enveloppé dès sa naissance, car on lui parle, et on le regarde dès le premier moment de sa vie, le petit nouveau né.

Cette prééminence du scopique avancée dans le miroir a été comme voilée par l'élargissement de la catégorie de l'imaginaire. A partir de L'instance de la lettre, Lacan (1957/1966) identifie l'imaginaire au signifié de la chaîne du langage, ce qui le fait dépendre fondamentalement d'autre chose que du visible - ça le fait dépendre du signifiantet, du coup, la fonction de l'image spéculaire a été éclipsée par le champ du signifié, par la signification et par le sens. Mais jusqu'où l'œil qui voit, le visible donc, dépend-t-il du signifiant ? L'image du miroir, qui est d'avant le sujet, est le seuil du monde visible, disait Lacan dans son texte de 1949. L'incidence propre du visible est restée comme une question en attente, avec la promotion, par Lacan, d'un imaginaire homologué au signifié de la chaîne signifiant. C'est cet imaginaire-là qui est subordonné à la chaîne du signifiant, tout comme la signification du phallus, signifiant du manque était posé dans cette première construction comme subordonné au signifiant du père. Mais la question se repose quand Lacan affirme que l'imaginaire n'est pas subordonné. Je vais y revenir.

Le premier instrument de l'escabeau, c'est l'image visible, mais les instruments sont multiples. Outre l'image arrangée dont je parlais, et ce sont aussi toutes les performances d'exception dans tous les domaines de la culture, à commencer par l'agriculture, mais aussi la science, les jeux du sport, et bien sûr l'art. Démonstration éminente par Joyce, mais s'il est une exception ce n'est pas parce qu'il a réussi à se faire un escabeau, mais par la manière de le faire. Comme dans mon Lacan lecteur de Joyce (2015). L'escabeau est le propre du " parlêtre », et il " est premier » selon Lacan, je l'ai déjà évoqué. Il reprend le narcissisme du miroir, mais il y ajoute cet autre narcissisme de l'invention. L'escabeau, c'est le miroir repensé et complété avec le narcissisme du désir, voire de la jouissance.

Si vous avez des doutes remettez sur le chantier les deux premières pages de la $2^{\mathrm{e}}$ Conférence Joyce le symptôme (1975a/2001) datée d'après le séminaire Le sinthome (1975-76/2005). Je dis chantier, car ça se lit non seulement mot à mot, mais phonème par phonème, et chacun de ces phonèmes étant écrits néologiquement, pour montrer que c'est la lettre graphique qui décide du sens à donner du son, comme le montre les diverses écritures du mot escabeau. Avec escabeau, même si vous ne parlez pas français, vous entendez trois sons, trois phonèmes qui dans nos langues n'ont pas de sens, et selon l'écriture leur sens change. Hessecabeau, le « h » est une lettre muette, ne s'entend pas, mais évoque le « h » de homme, tandis que le « esses » 
évoque le verbe " être ». Ou encore, "SKbeau », avec deux lettres alphabétiques, hors sens dans nos langues. Une seule équivoque graphique manque, notez-le, celle qui jouerait sur l'écriture du son «beau », qui pourrait s'écrire simplement de deux lettres alphabétiques. "b-a-ba », apprend-on à l'école primaire, «b-o-bo ", hors sens. Cette omission indique que Lacan a voulu garder la référence au côté scopique que comporte l'écriture «b, e, a u », «beau », pour désigner la belle forme du miroir dont il était parti 20 ans avant, celle que l'homme adore, comme il le dit, et c'est explicite quand il écrit "hissecroibeau ", où on entend résonner le narcissisme de l'image. Mais l'écriture « hissecroibeau » ajoute autre chose. Elle injecte le verbe se hisser - «oh hisse » dit-on en français, pour signifier l'effort qu'il faut pour hisser son image ou son nom de quelques degrés. Peut-on mieux dire la face laborieuse du narcissisme qui, loin de seulement se contempler, doit s'évertuer, ne pas se contenter du donné des images, mais se faire producteur en quelque chose. Et Lacan de l'expliciter : "LOM se lomellise à qui mieux mieux » (LACAN, 1975a/2001, p. 560); se lomelliser est néologique, n'existe pas en français, mais dit que l'homme, pour être homme, doit travailler à « se faire » homme. Pourquoi ça ? On ne sache pas en effet que dans le règne animal il y ait aucun équivalent que pour être tigre par exemple il faille se «tigrisser ». LOM doit se lomelliser parce qu'il vit de l'être (= vide l'être). Voilà, revenu, un thème des débuts, constante, c'est la parole qui introduit la question de l'être et qui du même mouvement le creuse d'un manque. C'est cet effet de la parole qui conditionne la nécessité de l'escabeau, le fait que la question de l'être soit posée pour le parlant. L'escabeau est donc le propre de l'homme, pas une caractéristique de seulement quelques-uns, et il est premier, commençant avec le miroir, mais allant au-delà, jusqu'à la promotion du nom. L'escabeau pour tous, mais quandmême, tous les escabeaux ne se valent pas du point de vue de la socialisation. Tout dépend des moyens et des produits.

Y a-t-il des figures de l'anti-escabeau. Celui qui a renoncé, par principe ou par accident, celui qui se désiste, qui renonce à se lomelliser. À voir en chaque cas, mais souvent trompeur. La preuve par la mégalomanie mélancolique: le sujet crie très fort qu'il ne vaut rien, mais tout aussi fort que nul ne l'égale à cet égard. Il se lomellise de sa nullité inégalable, et ce n'est pas à confondre avec les affects du manque d'auto-estime, comme on dit. Le manque d'auto-estime n'a pas plus de pouvoir causal que la plupart des affects, c'est patent, spécialement chez les femmes, qui souvent attestent de cet affect d'insuffisance et du sentiment de manque, voire d'impuissance, et ceci, d'autant plus qu'elles sont plus engagées dans la compétition phallique - autre nom de la compétition narcissique, mais qui, cependant, on le constate, ne quittent pas pour autant la table du jeu, voire emporte la mise - quoiqu'en tremblant, elles nous l'assurent souvent. Là, on les croit, mais à demi, car ce n'est qu'une mi-vérité, puisque la Vérité ne peut que se mi-dire. 


\section{Les trois narcissismes}

Pour ce qui est des moyens, j'ai évoqué, après le narcissisme de l'image, le narcissisme du désir, et puis il y a aussi celui de la jouissance. Ça en fait trois. Lacan a introduit le narcissisme du désir à propos de la relation entre les sexes, comme ce qui permet au fond de «se croire ", homme ou femme, et ça passe par le mode de relation au phallus qui, évidemment, suppose la parole. On peut élargir sa définition. Le narcissisme du désir, ça consiste à s'identifier à ce qui vous pousse dans la vie, sans que vous sachiez bien en général ce qui vous pousse - c'est ça le désir, et sa forme la plus remarquable, c’est de "ne pas céder sur son désir ", autrement dit, de tenir ferme sur votre être de désir, aussi opaque, voire déviant soit-il. Vous voyez l'ironie de l'histoire des analystes, car c'est justement ce que depuis le séminaire L'éthique de la psychanalyse (LACAN, 1959-60/1986), nous idéalisons comme le contraire du narcissisme. Or, Antigone, qui ne cède pas, c'est le narcissisme du sujet, autre façon de dire celui du désir, et, de fait, pour ce qui est de se faire un escabeau dans la mémoire des hommes, elle y a réussi, au prix de sacrifier tout ce qui relève des agréments de la vie. Et après le séminaire sur l'éthique, nous avons idéalisé avec Les quatre concepts fondamentaux de la psychanalyse (LACAN, 1964/1990), " la différence absolue » de fin d'analyse. Mais la différence absolue, c'est l'absolu de l'affirmation de soi, et sanctionnée par le désir de l'autre, l'analyste. Bref, que l'on ait pu continuer à dire après tout ça - ou à laisser entendre - qu'une analyse, d'une façon ou d'une autre, devait promouvoir un au-delà de l'aspiration à faire valoir son être propre, définition élargie que je retiens pour le narcissisme, faire valoir son être propre, relève d'une dénégation collective impressionnante, signe sans doute du refus de savoir que Lacan a diagnostiqué chez les analystes (LACAN, 1975b/2001).

Quand Lacan continuant d'avancer a évoqué "l'identification au symptôme ", et là, il s'agit de jouissance, ajoutant, en outre, que c'est plutôt court, quand il a parlé des unarités désassorties, là il y a eu, je ne dirais pas un réveil, mais au moins une inquiétude chez les analystes spécifiquement quant à l'ordre social, et aux relations à autrui. En effet, ce pas introduit une radicalisation.

Jusque-là, on pouvait méconnaître la menace que le narcissisme fait peser sur le lien social, carle désir étant désir de l'autre, le narcissisme du désir n'était pas forcément si asocial que cela. Avec le narcissisme de la jouissance, qui consiste à ne pas céder sur l'affirmation de sa modalité de jouissance et à s'y identifier - autrement dit, ne pas céder sur la préférence que chacun porte à la sienne propre -, eh bien, les choses changent, car la jouissance, contrairement au désir, n'est pas déterminée par l'Autre, le lieu du langage, mais par les accidents de la conjonction entre lalangue et le corps. J'ai personnellement déjà beaucoup insisté sur ce point, 
alors question: quid du pouvoir analytique de la parole de vérité toujours mi-dite sur les fixions de jouissance? Quid des sujets produits par l'analyse venue à sa fin ? Ne pourraient-ils pas être ces sujets identifiés à leur jouissance des sur-narcisses, cette fois aussi autosuffisants que Narcisse avant qu'il n'ait rencontré son image? Et quid de leur lien social, puisque la jouissance n'est pas liante?

Ce serait oublier que l'autosuffisance est impossible à qui parle comme tel, or même le narcissisme de l'escabeau vient de la parole. Le résultat, c'est qu'il n'y a pas moyen de se lomelliser tout seul. C'est ce que j'ai commencé à dire à Medel$\operatorname{lin}^{1}$. La jouissance relève du «Y a de l'Un», certes, mais dès lors que l'escabeau est premier et inévitable, il lui faut une cour comme au roi soleil lui-même. Pas moyen de se faire un escabeau sans l'autre. Le «m'as-tu-vu» donne à voir, c'est le paradigme du lien inter-narcissique, mais il y a d'autres façons; Joyce, comme on sait, est plutôt un « m'as-tu-lu »? Le comment est, donc, à étudier en chaque cas, pour les petites vies minuscules qui ont aussi leur escabeau, autant que pour les plus prééminentes. Tout ceci pour dire que la question des liens sociaux, au-delà du déclin des discours hiérarchisants, est ouverte. C'est le premier point. Cette révision suppose que l'on cesse d'opposer pulsions et narcissisme, comme on le fait généralement au nom de ceci que les pulsions quêtent quelque chose du côté de l'autre, sans majuscule, tandis que le narcissisme ne sortirait pas de son périmètre imaginaire. L'escabeau est plus que la construction d'image de soi, ce n'est pas simplement la statue érigée que Lacan évoquait au début, il n'est pas donné d'avance, il faut se le faire. Comment le « se faire " de la pulsion (puisque c'est la formule par laquelle Lacan définit la pulsion) contribue-t-il à l'érection de l'escabeau est évidemment une question. Si, comme le dit Lacan, LOM, il fait payer une dîme à l'autre, c'est bien qu'il sort du périmètre de son image scopique, et les pulsions, si vous m'avez suivie, sont à inclure dans ce narcissisme élargi, tout autant qu'elles le sont d'ailleurs dans l'amour en général et dans toutes relation d'objet. « En toi plus que toi » disait la fin du Séminaire XI (LACAN, 1964/1990), eh bien, avec le narcissisme de l'escabeau, il faut ajouter « en moi, plus que moi ». Autrement dit, c'est un narcissisme qui doit compter avec l'objet $a$, l'invisible comme je l'appelle, qui manque à toute jouissance. L'image est première, mais pour le parlant elle est chasuble, enveloppe de l'objet soustrait, et ça s'applique à l'image de soi aussi bien qu'à celle de l'autre.

Reste la question de l'autonomie de l'imaginaire avancée avec le nœud borroméen, elle engage celle de l'autonomie du visible, puisque l'image scopique en est

1 Référence au IXe Rendez-vous de I'Internationale des Forums et Ve Rencontre de l'École de Psychanalyse des Forums du Champ Lacanien, qui a eu lieu du 14 au 17 juillet 2016 à Medellín (Colombie). 
le noyau. Qu'est-ce qui conduit Lacan à récuser la subordination de l'imaginaire au symbolique? C'est sa redéfinition du symbolique, disons de l'inconscient, elle est explicite, " contrairement à ce que j'ai dit ", l'inconscient n'est pas une chaîne signifiant. Qu'est-il ? Une série, pas une chaîne, mais une suite numérique, une suite de signifiantsqui sont autant d'unités numériques, chacune solidaire de l'objet $a$ qui manque. Ce changement de définition tient à ce que Lacan, s'étant interrogé au-delà de la chaîne de la parole sur la relation du sujet au signifiant (sujet entré dans le réel), a produit la formule toujours répétée, le signifiant est «ce qui représente le sujet " pour un autre signifiant. Eh bien, l'inconscient ce sont les signifiantsqui ne représentent pas le sujet, qui viennent de lalangue et qui affectent son corps. Ce sont les élaboration de D'un Autre à l'autre (LACAN, 1968-69/2006) et des textes périphériques, où il formule l'inconscient est un "savoir sans sujet ». Je ne rappelle succinctement ce virage que pour indiquer qu'il fonde le changement d'affirmation sur l'ancienne idée d'une subordination de l'imagináire à la chaîne, et qu'il repose donc la question du poids de l'image en elle-même.

J'ai laissé la question en suspens, mais je crois qu'elle était là pour Lacan. J'en veux pour preuve les développements de 1964 sur l'œil et le regard dans le Séminaire XI (LACAN, 1964/1990) à l'occasion de la mort de Merleau Ponty. Il l'élabore de façon nouvelle, et quand il note que nous sommes des êtres regardés, que le monde est omni voyeur, ce n'est pas pour affirmer une paranoïa généralisée, mais bien une prévalence spécifique du registre du visible, et ouvrir la question de sa relation à la division du sujet.

Mais au-delà même du sémináire Le sinthome, Lacan (1975-76/2005), curieusement, repose la question: pourquoi l'homme est-il si infatué à son image ? Je dis curieusement, car on pourrait penser qu'il avait la réponse. Il en a donné deux, je l'ai dit, celle de la prématuration, puis celle de la division du sujet, de l'objet qui manque à la complétude et que l'image recouvre. Qu'il repose la question me laisse à penser que ces réponses-là ne lui paraissaient pas encore suffisantes. Ce point importe, car il a un enjeu : celui de l'évaluation des variantes de l'escabeau.

\section{Références bibliographiques}

FREUD, S. (1914) Introducción del narcisismo. In: Obras Completas de Sigmund Freud. Buenos Aires: Amorrortu, 1985, v. 14.

LACAN, J. (1938) Les complexes familiaux dans la formation de l'individu. Essai d'analyse d'une fonction en psychologie. In: Autres écrits. Paris: Seuil, 2001. . (1946) Propos sur la causalité psychique. In: Ecrits. Paris: Seuil, 1966. . (1949) Le stade du miroir comme formateur de la fonction du Je. In: Ecrits. Paris: Seuil, 1966. 
. (1953) Fonction et champ de la parole et du langage enpsychanalyse. In:

Ecrits. Paris: Seuil, 1966.

. (1957) L'instance de la lettre dans l'inconscient ou la raison depuis

Freud. In: Ecrits. Paris: Seuil, 1966.

. (1958) La direction de la cure et les principes de son pouvoir. In: Ecrits.

Paris: Seuil, 1966.

. (1959-60) Le séminaire, livre 7: l'éthique de la psychanalyse. Paris: Seuil, 1986.

. (1960a) Propos directifs pour un congrès sur la sexualité féminine.

Ecrits. Paris: Seuil, 1966.

. (1960b) Remarque sur le rapport de Daniel Lagache: "psychanalyse et

structure de la personnalité”. In: Ecrits. Paris: Seuil, 1966.

. (1964) Le séminaire, livre XI: les quatre concepts fondamentaux de la psychanalyse. Paris: Seuil, 1990.

. (1968-69) Le séminaire, livre XVI: d'un Autre à l'autre. Paris: Seuil, 2006.

. (1972) L'étourdit. In: Autres écrits. Paris: Seuil, 2001.

. (1975a) Joyce, le Symptôme. In: Autres écrits. Paris: Seuil, 2001.

. (1975b) Introduction à l'édition allemande d'un premier volume des

Écrits. In: Autres écrits. Paris: Seuil, 2001.

. (1975-76) Le séminaire, livre XXIII: le sinthome. Paris: Seuil, 2005.

OVÍDIO. Les Métamorphoses. Paris: Gallimard, 1992.

SOLER, C. Lacan, lecteur de Joyce. Paris: PUF, 2015.

WILDE, O. Collected works of Oscar Wilde. London: Routledge, 1993.

\section{Nouvelle économie du narcissisme New economics of narcissism}

\section{résumé}

Ce texte indique un renouvellement du concept de narcissisme des changements introduits par Lacan, les trottoirs dans l'avènement de la théorie du nœud borroméen. Pour cela, l'auteur montre comment l'enregistrement imaginaire a été examiné par Lacan à sa subordination à la symbolique. Reprise du stade du miroir, qui souligne les conséquences de cette nouvelle interprétation du concept de narcissisme, établissant trois narcissismes: l'image, le désir et le plaisir. Ainsi, l'auteur propose une nouvelle économie du narcissisme à travers le mythe de Narcisse, relectures d'une étude sur l'escabeau, où la dimension de l'imagination est élargie avec l'inclusion de l'objet. Cette conception a un impact considérable sur la question des liens sociaux aujourd'hui. 


\section{mots-clés:}

Narcissisme; stade du miroir; imaginaire; escabeau.

\section{abstract}

This text indicates a renewal of the concept of narcissism as of the changes introduced by Lacan, based on the advent of the Borromean node theory. For this, the author demonstrates how the imaginary record was reviewed by Lacan regarding its subordination to the symbolic. Resuming the Mirror Stage, she points out the consequences of this new reading on the concept of narcissism, establishing three narcissisms: the ones of image, desire and joy. Thus, the author proposes a new economy of narcissism through the re-reading of the myth of Narcissus, of a study on the stool, in which the imaginary dimension is enlarged with the inclusion of the object a. This conception has considerable effects on the issue of current social ties.

\section{keywords:}

Narcissism; mirror stage; imaginary; escabeau.

\section{Recebido:}

30/05/2017

\section{Aprovado:}

01/07/2017 